This item was submitted to Loughborough's Research Repository by the author.

Items in Figshare are protected by copyright, with all rights reserved, unless otherwise indicated.

\title{
Laser vibrometry: pseudo-vibrations
}

PLEASE CITE THE PUBLISHED VERSION

http://dx.doi.org/10.1016/0022-460X(89)90705-0

PUBLISHER

(c) Elsevier

VERSION

AM (Accepted Manuscript)

LICENCE

CC BY-NC-ND 4.0

REPOSITORY RECORD

Rothberg, Steve, J.R. Baker, and Neil A. Halliwell. 2019. "Laser Vibrometry: Pseudo-vibrations". figshare. https://hdl.handle.net/2134/9647. 
This item was submitted to Loughborough's Institutional Repository (https://dspace.lboro.ac.uk/) by the author and is made available under the following Creative Commons Licence conditions.

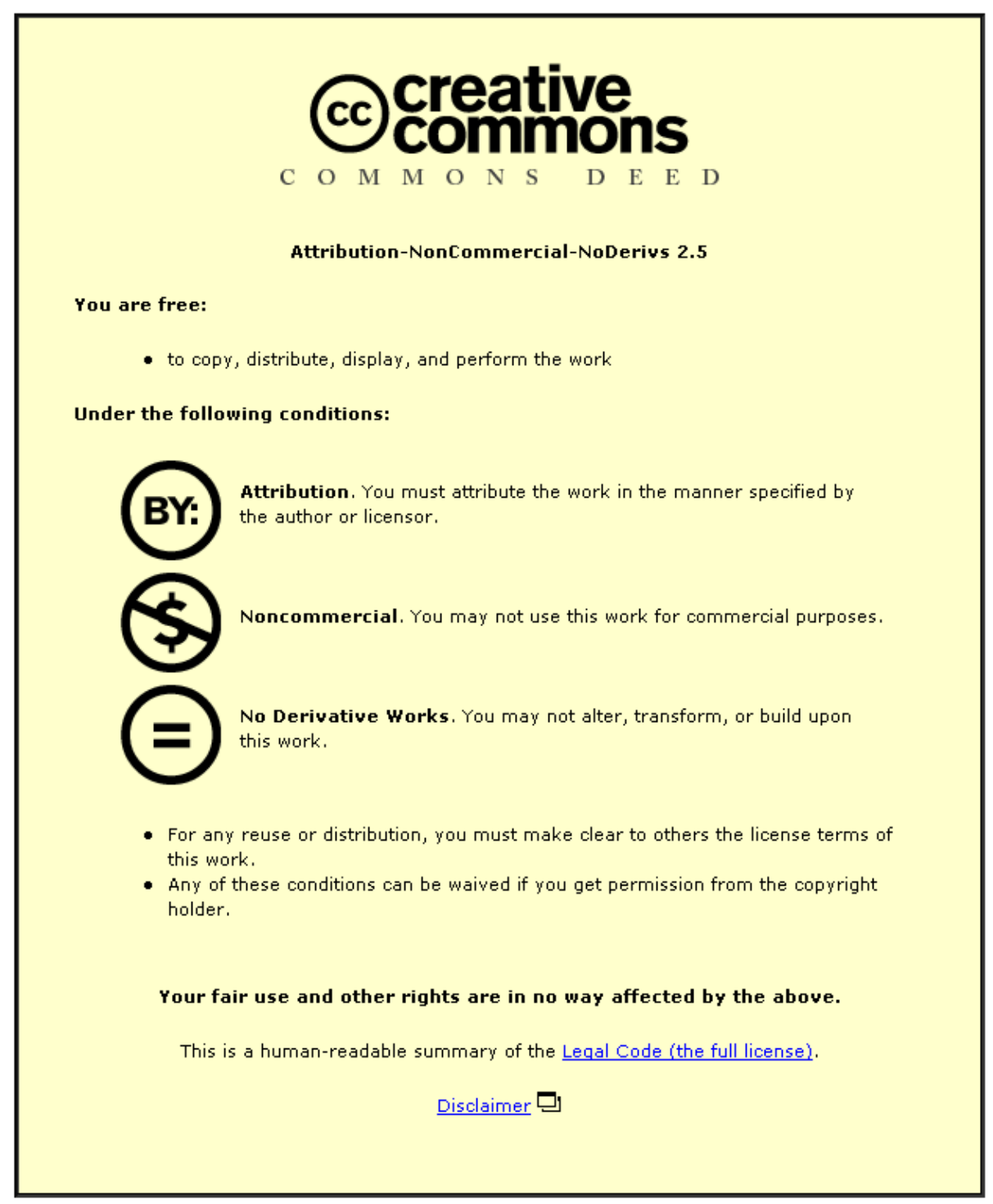

For the full text of this licence, please go to: http://creativecommons.org/licenses/by-nc-nd/2.5/ 
LETTER TO THE EDITOR

IASER VIBROMETRY: PSUEDO-VIBRATIONS

S.J.Rothberg, J.R.Baker, N.A.Halliwell

ISVR, University of Southampton,

Highfield,

Southampton SO9 $5 \mathrm{NH}$,

ENGLAND.

9 Pages 5 Figures 0 Tables 
LASER VIBROMETRY: PSUEDO-VIBRATIONS

\author{
S.J.Rothberg, \\ ISVR, \\ University of Southampton, \\ Highfield, \\ Southampton SO9 5NH, \\ ENGLAND .
}




\section{INTRODUCTION}

The application of Laser Doppler Velocimetry (LDV) to the measurement of normal-to-surface vibration of a solid surface is now established as a technique complementary to the use of an accelerometer. Several practical systems have been developed and a number are now commercially available. Each velocimeter relies on the same principle of operation, namely the detection of a Doppler shift, $f_{D}$, in the light scattered from a vibrating target. Fig. 1 shows a typical vibrometer arrangement. Since the photodetector cannot respond quickly enough to detect the light frequency directly, scattered light from the vibrating surface is mixed with a reference beam and heterodyned on the detector surface. In addition, in order to resolve the sign of the vibration velocity, it is necessary to pre-shift the reference beam by a known amount, $f_{R}$, resulting in an optical beat at the detector of frequency $\left(f_{R} \pm f_{D}\right)$. An appropriate Doppler signal processor then demodulates the detector signal to produce a time-resolved analogue of the target vibration velocity (in the direction of the incident beam). Systems differ in the method adopted to produce the reference beam frequency shift. Bragg cells [1], diffraction gratings [2] rotating scattering discs [3] and frequency modulation of the laser beam itself [4] have all been used successfully.

In a thorough treatment of the heterodyne process, however, it is very important to consider the effects of the formation of a speckle pattern in light backscattered from the target surface. A speckle pattern is produced when component wavelets of the incident laser beam are dephased on scattering from a surface that is rough on the scale of the optical wavelength. This includes most surfaces of engineering interest. Constructive and destructive interference of these dephased but still coherent wavelets results in a chaotic distribution in backscatter of high and low intensities referred to as a "speckle pattern". The properties of the latter are best described statistically. Speckle intensities have a negative exponential probability distribution whereas their phases are distributed uniformly between 0 and $2 \pi$ rads [5]. For a normally incident laser 
beam, translation of the target surface in some non-normal direction (e.g. tilt, in-plane motion or rotation) will cause the speckles to either "boil", move in sympathy with the surface or perform some combination of these two [6]. Whatever the motion type, changes in the spatial characteristics of the speckle pattern on the detector will cause consequent changes in the amplitude and phase of the detector output. For periodic non-normal motion this letter shows how spurious information is introduced into the output spectrum which cannot be distinguished from genuine normal-to-surface vibration information; hence the name "psuedo-vibration". The implications of this phenomenon are twofold. Firstly, data generated using laser vibrometers requires careful interpretation and, secondly, noise-floors for this type of instrumentation cannot be specified without reference to the particular target surface dynamics.

\section{THEORY OF OPERATION}

For convenience, consider the case where the reference beam contribution has uniform amplitude $\left(E_{R}\right)$ and phase $\left(\varphi_{R}\right)$ across the detector and has undergone a constant frequency shift $\left(\omega_{\mathrm{R}}\right)$. Hence, neglecting polarisation effects:

$E_{R}(t)=E_{R} \cos \left[\left(\omega+\omega_{R}\right) t+\varphi_{R}\right]$

where $\omega$ is the laser light frequency. The output of the photodetector may be predicted from the phasor addition of the contributions from the target and reference beams in each element of area and subsequent integration over the entire active area. For a stationary target surface the contribution of the $p^{\text {th }}$ speckle on the detector from the target may be written:

$\mathrm{E}_{\mathrm{Tp}}(t)=\mathrm{E}_{\mathrm{Tp}} \cos \left[\omega t+\varphi_{\mathrm{Tp}}\right]$ 
where $\mathrm{E}_{\mathrm{T} p}$ and $\varphi_{\mathrm{Tp}}$ are, respectively, the amplitude and phase of the $\mathrm{p}^{\text {th }}$ target speckle. If the target surface vibrates sinusoidally, the speckle phase term becomes modulated by the displacement and equation (2) is re-written:

$\mathrm{E}_{\mathrm{Tp}}(t)=\mathrm{E}_{\mathrm{Tp}} \cos \left[\omega t+\varphi_{\mathrm{Tp}}-2 k \mathrm{a}_{\mathrm{v}} \sin \omega_{\mathrm{v}} \mathrm{t}\right]$

where $a_{\mathrm{V}}$ and $\omega_{\mathrm{V}}$ are the target displacement amplitude and frequency, respectively, and $k$ is the light wavenumber. Summing the reference beam and target beam contributions and neglecting d.c. terms yields the a.c. component of the resultant intensity as:

$I_{p}(t)=2\left(I_{T p} I_{R}\right)^{1 / 2} \cos \left[\left(\omega_{R} t+2 k a_{v} \sin \omega_{v} t\right)+\left(\varphi_{R}-\varphi_{T p}\right)\right]$

where $I_{R}$ and $I_{T p}$ are the intensities of the reference beam and the $p^{\text {th }}$ target beam speckle, respectively. Assuming each speckle to have constant phase and intensity over its extent, the component, $i_{p}(t)$, of the photodetector output, due to interference of the $\mathrm{p}^{\text {th }}$ target beam speckle and the frequency shifted reference beam is then given by:

$i_{p}(t)=S A_{p} I_{p}(t)$

where $S$ is the radiant sensitivity of the photodetector and $A_{p}$ is the area of the speckle incident on the detector. For $\mathrm{P}$ speckles incident on the detector the total photodetector output may be expressed in the form:

$i(t)=\sum_{p=1}^{P} i_{p}(t)=S I_{\text {res }} \cos \left[\left(\omega_{R} t+2 k a_{v} \sin \omega_{v} t\right)+\Phi_{r e s}\right]$

where: 
$I_{\text {res }}=2\left(I_{R}\right)^{1 / 2}\left[\sum_{p=1}^{P} \sum_{q=1}^{P} A_{p} A_{q}\left(I_{T p} I_{T q}\right)^{1 / 2} \cos \left(\varphi_{T p}-\varphi_{T q}\right)\right]^{1 / 2}$

and

$\tan \left[\Phi_{\text {res }}\right]=\left[\begin{array}{ccc}\sum_{p} & A_{p}\left(I_{p}\right)^{1 / 2} \sin \left(\varphi_{T p}-\varphi_{R}\right) \\ \frac{p=1}{P} A_{p}\left(I_{p}\right)^{1 / 2} \cos \left(\varphi_{T p}-\varphi_{R}\right)\end{array}\right]$

Note from equation (7) how combinations of speckles such that:

$\left(\varphi_{\mathrm{Tp}}-\varphi_{\mathrm{Tq}}\right)=(2 \mathrm{n}+1) \Pi / 2 \quad$ where $\mathrm{n}$ is any +ve integer

may lead to problems due to low signal amplitudes. If the $P$ target beam speckles on the detector do not change in either phase, amplitude or position (relative to the detector) during the course of a measurement then $I_{\text {res }}$ and $\Phi_{\text {res }}$ are constant in time and the measured beat frequency, $\omega_{D}$, given by the time derivative of the argument of the cosine term in equation (6) i.e.

$\omega_{\mathrm{D}}=\omega_{\mathrm{R}}+2 k \mathrm{a}_{\mathrm{V}} \omega_{\mathrm{V}} \cos \omega_{\mathrm{V}} t+\frac{\mathrm{d} \Phi_{\mathrm{res}}}{\mathrm{dt}}$

is equal to the required value of:

$\omega_{D}=\omega_{R}+2 k a_{v} \omega_{V} \cos \omega_{v} t$

since $\left(d \Phi_{\text {res }} / d t\right)=0$. In this case, neglecting electronic noise, the output spectrum of the instrument will consist of a single peak at $\left(\omega_{\mathrm{v}} / 2 \Pi\right) \mathrm{Hz}$. However, if the spatial characteristics of the speckle pattern on the detector change then, performing the summation of equation (8) over a changing population of 
randomly-phased speckles introduces time dependancy into $\Phi_{\text {res }}$ and leads to a non-zero and changing value of $\left(\mathrm{d} \Phi_{\text {res }} / \mathrm{dt}\right)$. In this case, the output spectrum of the instrument will consist of a peak at $\left(\omega_{\mathrm{V}} / 2 \pi\right) \mathrm{Hz}$ and spurious noise corresponding to the frequency content of ( $d \Phi_{\text {res }} / \mathrm{d} t$ ). Moreover, when the speckle pattern changes are induced by non-normal target motions which, in general, will be periodic with the same fundamental frequency as the normal-to-surface vibration, ( $\mathrm{d} \Phi_{\text {res }} / \mathrm{dt}$ ) takes a psuedo-random form with a fundamental frequency also equal to $\left(\omega_{\mathrm{v}} / 2 \Pi\right) \mathrm{Hz}$. The characteristic spectrum of a psuedomrandom signal consists of approximately equal amplitude peaks at the fundamental frequency and subsequent harmonics. Thus, overall, the output spectrum of the instrument will consist of peaks at frequencies given by:

$f=n \frac{\omega_{v}}{2 \Pi} \quad$ where $n=1,2, \ldots \ldots \ldots, \infty$

and the spurious information (psuedo-vibration) will be indistinguishable from the genuine vibration information. The magnitude of each harmonic noise peak will depend on the amplitude and frequency of fluctuations in (d $\Phi_{\text {res }} / \mathrm{dt}$ ) which, in turn, will depend on the amplitude of the non-normal motion and the detector and speckle size involved.

\section{SOURCES OF PSUEDO-VIBRATION}

Three primary target motion types are capable of inducing the necessary speckle pattern changes: tilt, in-plane motion and rotation. The consequent effects on the speckle pattern may be explained in terms of Fourier optics, whereby light in the (far-field) observation plane (detector) is found to be proportional to the spatial Fourier transform of the illumination plane (target), according to the Fraunhofer assumption [7]. If, in the absence of any surface motion, the field in the target plane is given by $E\left(x_{t}, y_{t}\right)$ and that in the detector plane by $E\left(x_{d}, y_{d}\right)$ 
then:

$E\left(x_{d}, y_{d}\right) \propto F T\left\{E\left(x_{t}, y_{t}\right)\right\}$ evaluated at frequencies $\left(x_{d} / \lambda z\right),\left(y_{d} / \lambda z\right)$

where $\lambda$ is the laser wavelength and $z$ is the target-detector distance. The effects of tilt and lateral motion may now be predicted using the shift theorem of Fourier transforms. To model a small two-dimensional tilt of amplitude $(\alpha, \beta)$ rads the original target plane field is simply pre-multiplied by a phase factor which may be written:

$\mathrm{FT}\left\{\exp \left[-\mathrm{j} 2 \mathrm{k}\left(\alpha \mathrm{x}_{\mathrm{t}}+\beta \mathrm{y}_{\mathrm{t}}\right)\right] \mathrm{E}\left(\mathrm{x}_{\mathrm{t}}, \mathrm{y}_{\mathrm{t}}\right)\right\} \alpha \mathrm{E}\left(\mathrm{x}_{\mathrm{d}}+2 \alpha z, \mathrm{y}_{\mathrm{d}}+2 \beta \mathrm{z}\right)$

Therefore, tilt of the target produces an in-plane displacement of the detector plane speckle pattern equivalent to $2 \alpha z$ and $2 \beta z$ in the $\mathrm{x}$ and $\mathrm{y}$ directions respectively. The speckle pattern translates across the detector surface and the changing population of speckles produces amplitude and phase modulation of the detector output. Conversely, a two dimensional in-plane translation of the target of amplitude $(\mathrm{a}, \mathrm{b})$ metres will impose a linear phase ramp through the detector plane speckle pattern given by:

FT $\left\{E\left(x_{t}-a, y_{t}-b\right)\right\} \alpha \exp \left[-j(2 \pi / \lambda z)\left(a x_{d}-b y_{d}\right)\right] E\left(x_{d}, y_{d}\right)$

In practice, therefore, for small in-plane translations, amplitude and phase modulation of the detector output occurs through phase changes of the target speckles on its active area. Larger in-plane translations involving significant changes in the illuminated population of scatterers will begin to cause decorrelation of the speckle pattern referred to as speckle "boiling" [6]. In this case, amplitude and phase modulation of the detector output again occurs through the continuous change in the population of speckles on the detector. 
The more complex case of rotation may be considered as a combination of the above two effects where amplitude and phase modulation of the detector output again occur through the continuous change in the speckle pattern incident on the detector.

\section{EXPERIMENTAL RESULTS}

The following results were generated using the vibrometer arrangement shown in figure 1 .

Figure 2 shows a laser vibrometer measurement of norma1-to surface vibration taken from an electrodynamic shaker excited at $50 \mathrm{~Hz}$. The form of the spectrum is typical of laser vibrometer measurements on periodically excited structures. Figures 3a, 4 and 5 show examples of the psuedo-vibration phenomenon under conditions of nominally zero normal-to-surface motion.

In figures $3 \mathrm{a}, \mathrm{b}$ a comparison is shown of vibrometer and accelerometer measurements taken from a surface translating in-plane. A cylindrical bar running in a high tolerance bearing was attached at one end to a shaker. The target laser beam was incident on the circumference of the bar perpendicular to the motion of the latter and the accelerometer was mounted on the fixed bearing. Vibration levels as measured by the vibrometer are some $30 \mathrm{~dB}$ higher than those measured using the accelerometer, indicating the extent to which measurements may be affected by speckle motions.

Figure 4 represents the vibrometer output from a surface undergoing a pure tilt. This was achieved by connecting the cylindrical bar in the above rig via a crank arrangement to the shaker which displaced the bar angularly back and forth about its central axis. The target for the incident laser beam was a machined "flat" on the bar coincident with a plane containing the rotational axis. In this way it 
was possible to obtain the necessary pure tilt with negligible in-plane or normal-to-surface motion. Here again, the spectrum contains energy at the fundamental motion frequency and higher order harmonics.

Figure 5 shows the vibrometer output from a target rotating at constant speed. The now familiar pattern of psuedo-vibration peaks at the fundamental frequency and subsequent harmonics is again apparent. However, in this instance, the peaks do not display the same amplitude "roll-off" as the previous spectra, remaining approximately constant in amplitude with some random fluctuation. This is typical of measurements under conditions where a large number of changes occur in the speckle pattern on the detector. As such, a spectrum of this form may be more easily attributed to speckle motions than to true vibration unlike the aforementioned cases of in-plane motion and tilt.

It is clear from these figures how quoted noise-floors for commercial instruments are invalidated under common target motion conditions found in practice. In each of the psuedo-vibration cases demonstrated energy is distributed at the fundamental motion frequency and subsequent harmonics and, as such, the form of the resultant spectra is not readily distinguishable from the true case shown in figure 2. In addition, there is a significant risk that falsely high levels may be recorded as shown in figures $3 a, b$.

\section{CONCLUSIONS}

It has been demonstrated how the spectra of laser vibrometer measurements may contain surface motion information unrelated to normal-to-surface vibrations. In particular, since this spurious information will usually appear at the fundamental vibration frequency and subsequent harmonics, care is required in the interpretation of measurement data where genuine vibration data may be indistinguishable from speckle-induced psuedo-random noise. These phenomena show 
that it is not possible to determine the noise-floor of this type of instrumentation without specific reference to the target surface dynamics. In general it is clear that, at low vibration levels, interpretation of laser vibrometer measurements will require a degree of engineering judgement. Further work is continuing to quantify these effects, particularly for the case of vibrometer measurements on rotating targets.

\section{REFERENCES}

[1] P.BUCHAVE 1975 Optics and Laser Technology 7, 11-16, Laser Doppler Velocimeter with Variable Optical Frequency Shift.

[2] J.OLDENGARM, A.H.VON KRIEKEN and H.RATERINK 1973 Optics and Laser Technology 5, 249-252 Laser Doppler Velocimeter with Optical Frequency Shifting

[3] C.J.D.PICKERING, N.A.HALLIWELL and T.H.WILMSHURST 1986 Journal of Sound and Vibration $107(3), 471-485$, The Laser Vibrometex: A Portable Instrument

[4] R.I.LAMING, M.P.GOLD, D.M.PAYNE and N.A.HALLIWELL 1986 Electronics Letters 22, 167-168, Fibre-Optic Vibration Probe

[5] J.C.DAINTY 1975 Laser Speckle and Related Phenomena, Bexlin: Springer-Verlag [6] N.TAKAI, T.IWAI and T.ASAKURA 1983 Applied optics, 22(1), 170-177, Correlation Distance of Dynamic Speckles

[7] J.W.GOODMAN 1968 Introduction to Fourier Optics, London: McGraw-Hill 


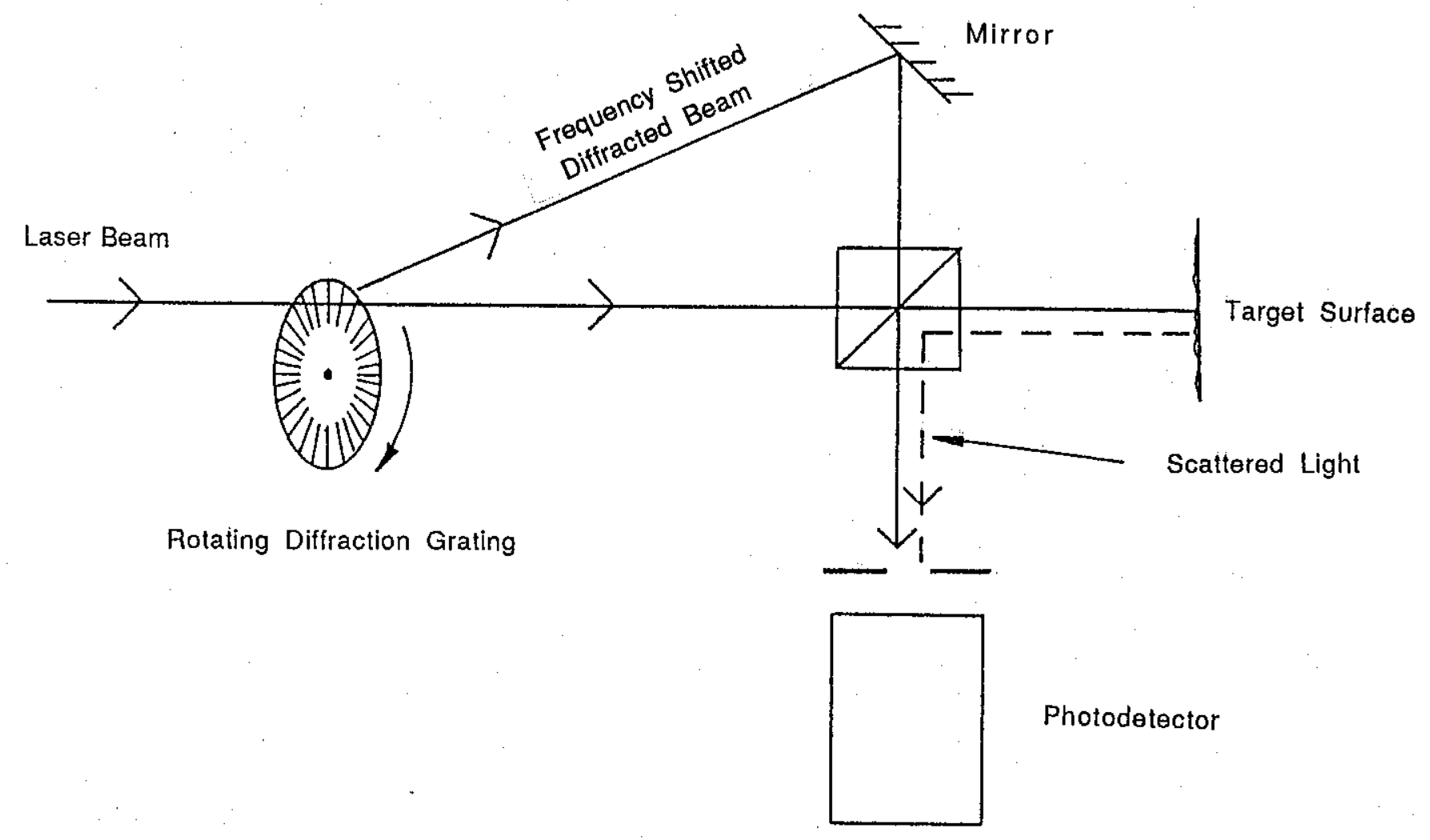

Figure 1. Optical geometry of a typical laser vibrometer. 


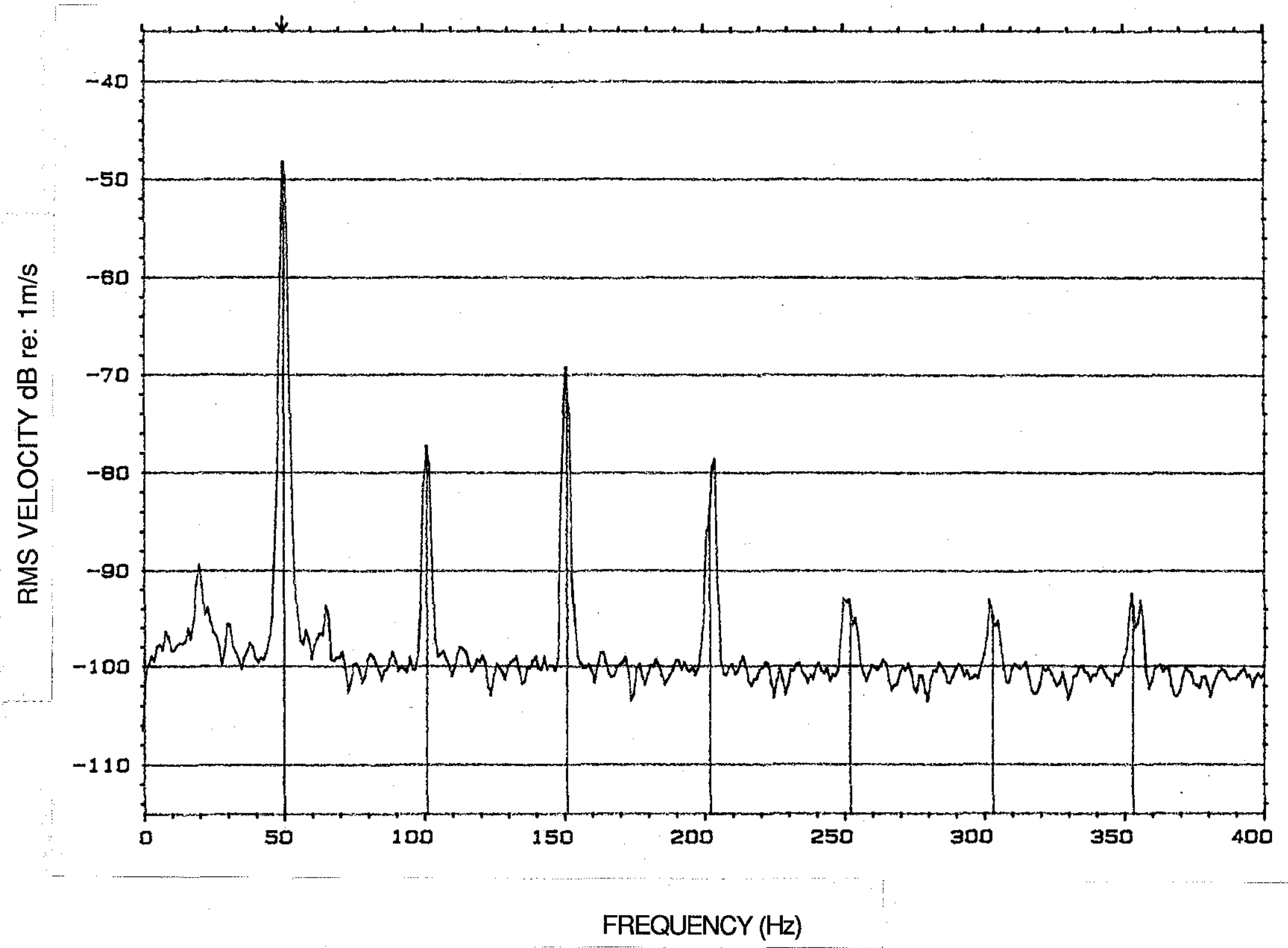

Figure 2. Vibrometer output from a normal-to-surface vibration. 


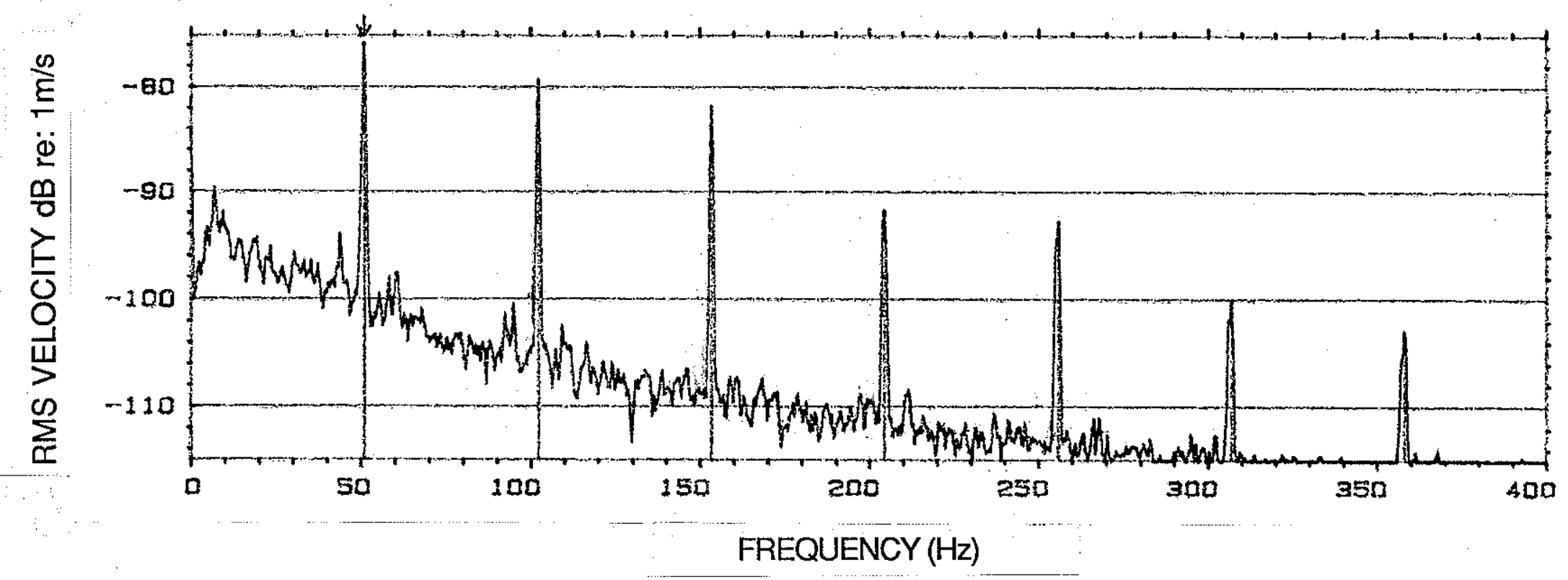

Figure 3. (a) Vibrometer output from an in-plane vibration

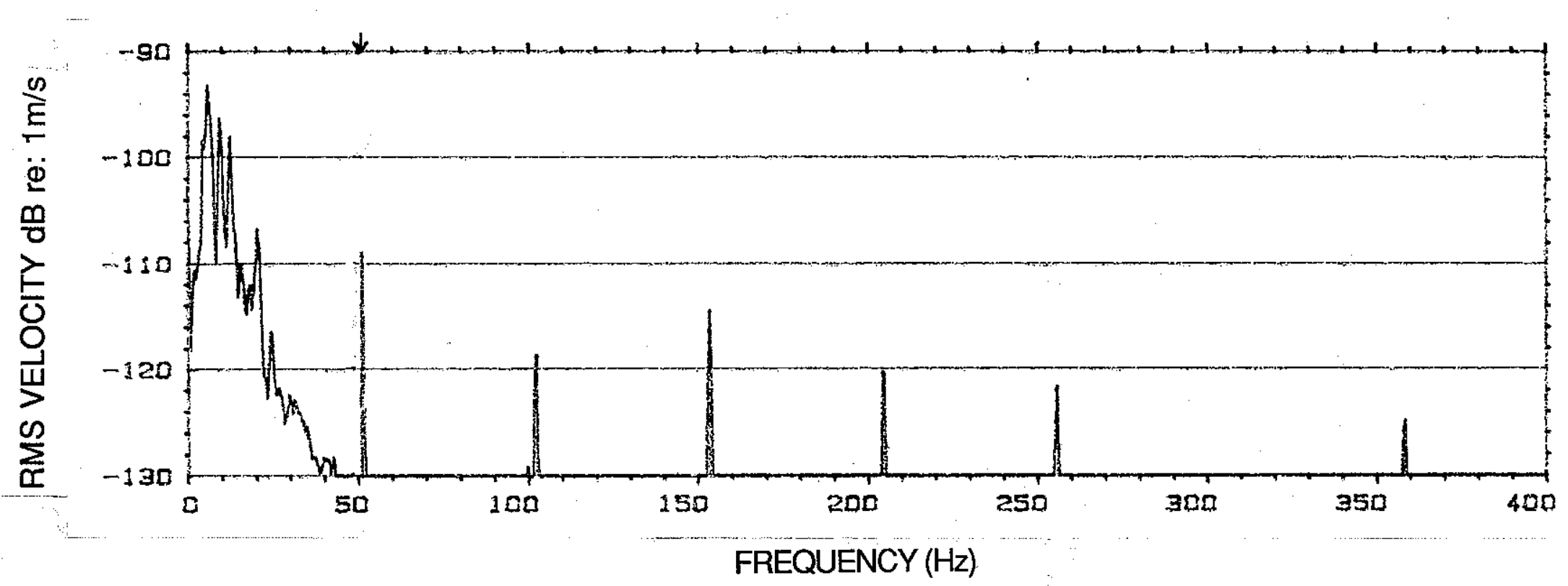

Figure 3.(b) accelerometer measurement of true normal-to-surface vibration. 


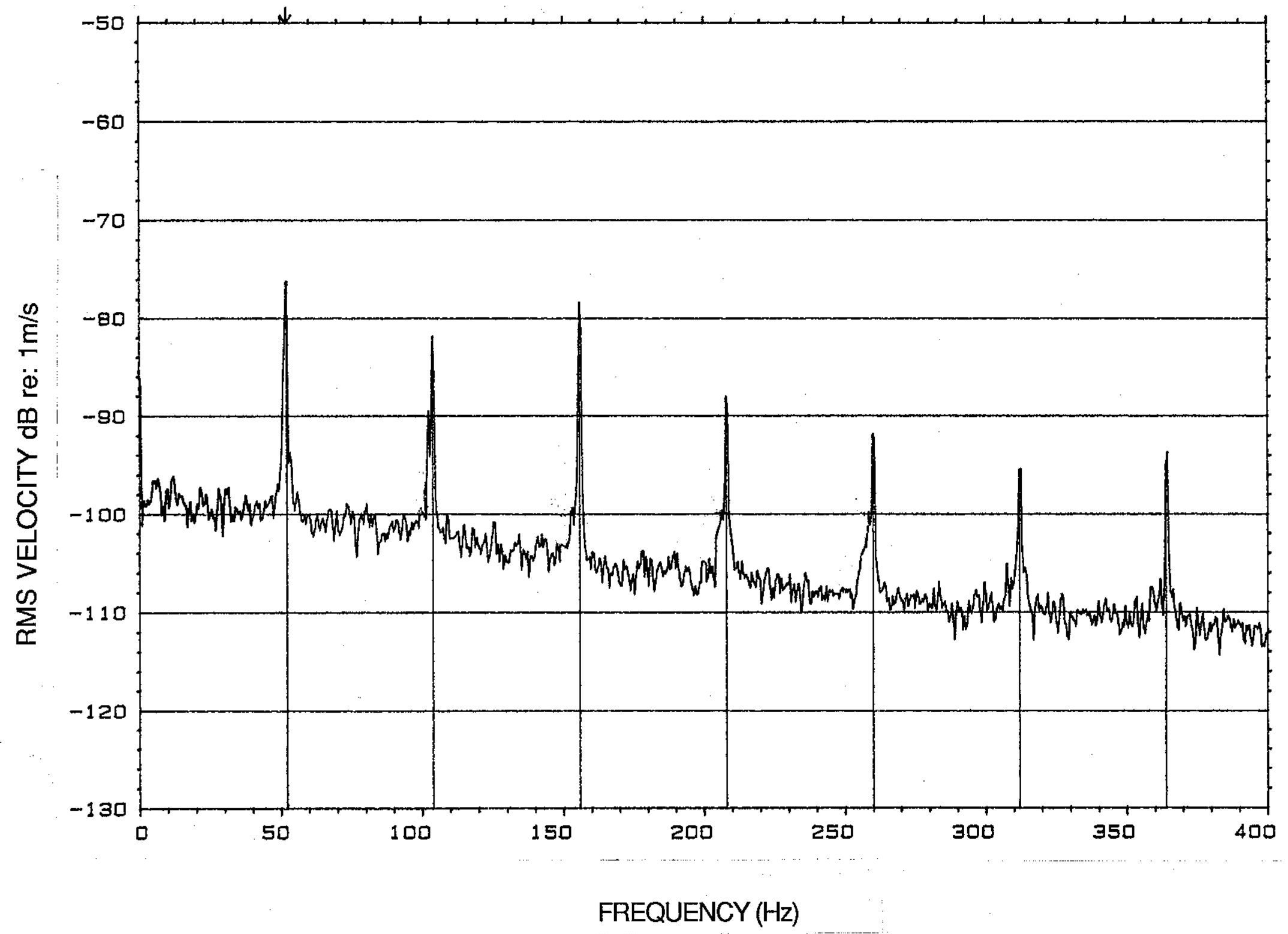

Figure 4. Vibrometer output from a tilting surface. 


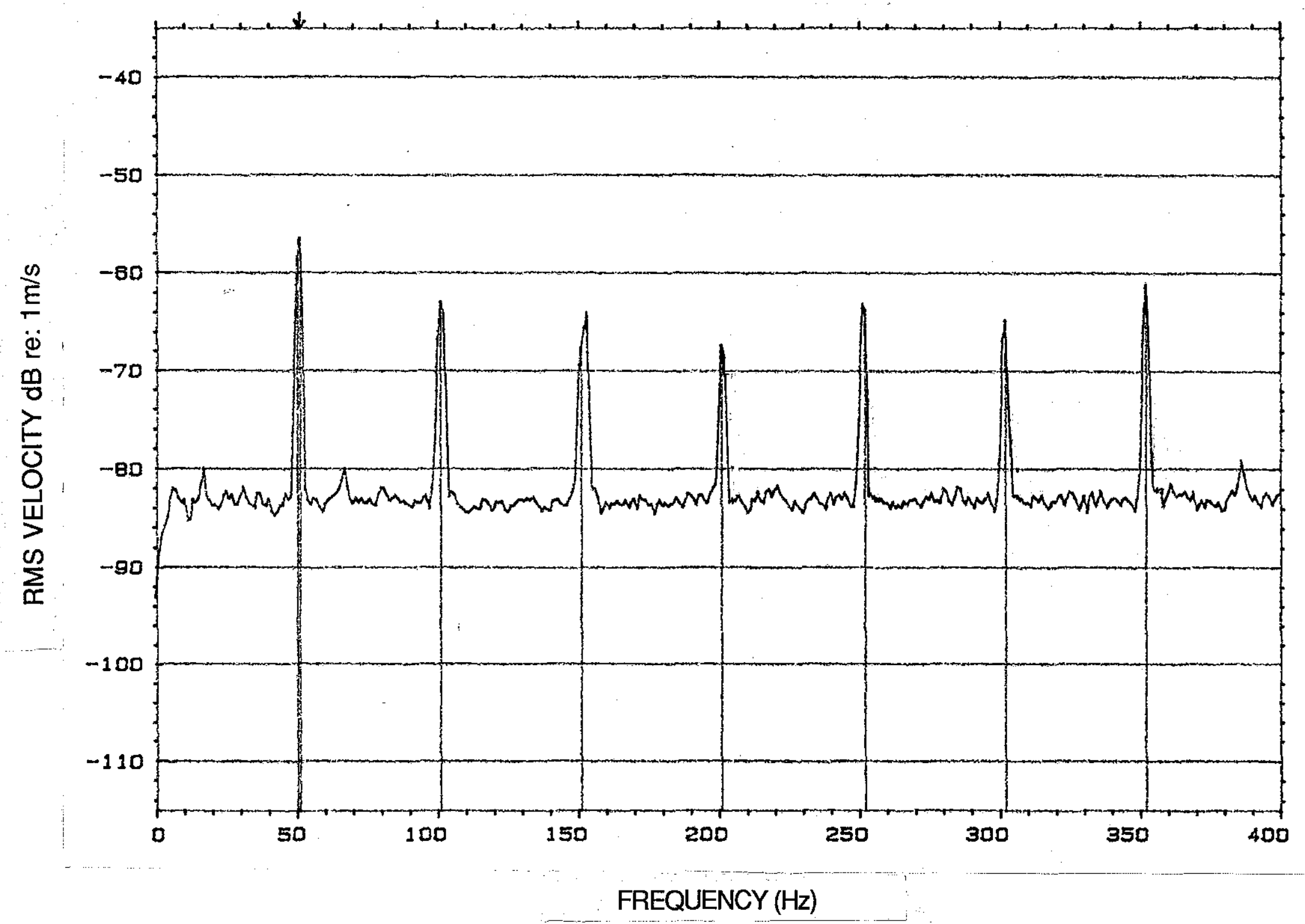

Figure 5. Vibrometer output from a rotating surface. 